\title{
Ultralow-power spectroscopy of a rare-earth spin ensemble using a superconducting resonator
}

\author{
P. Bushev, ${ }^{1}$ A. K. Feofanov, ${ }^{1,6}$ H. Rotzinger, ${ }^{1}$ I. Protopopov, ${ }^{2}$ J. H. Cole, ${ }^{3,4}$ C. M. Wilson, ${ }^{5}$ G. Fischer, ${ }^{1}$ \\ A. Lukashenko, ${ }^{1}$ and A. V. Ustinov ${ }^{1,6}$ \\ ${ }^{1}$ Physikalisches Institut, Karlsruhe Institute of Technology, D-76128 Karlsruhe, Germany \\ ${ }^{2}$ Institut für Nanotechnologie, Karlsruhe Institute of Technology, D-76021 Karlsruhe, Germany \\ ${ }^{3}$ Institut für Theoretische Festkörperphysik, Karlsruhe Institute of Technology, D-76128 Karlsruhe, Germany \\ ${ }^{4}$ Applied Physics, School of Applied Sciences, RMIT University, Melbourne 3001, Australia \\ ${ }^{5}$ Microtechnology and Nanoscience, MC2, Chalmers University of Technology, SE-412 96 Göteborg, Sweden \\ ${ }^{6}$ DFG-Center for Functional Nanostructures (CFN), D-76128 Karlsruhe, Germany \\ (Received 17 February 2011; revised manuscript received 6 July 2011; published 5 August 2011)
}

\begin{abstract}
Interfacing superconducting quantum processors, working in the $\mathrm{GHz}$ frequency range, with optical quantum networks and atomic qubits is a challenging task for the implementation of distributed quantum information processing as well as for quantum communication. Using spin ensembles of rare-earth ions provides an excellent opportunity to bridge microwave and optical domains at the quantum level. In this Rapid Communication, we demonstrate the ultralow-power, on-chip, electron-spin-resonance spectroscopy of $\mathrm{Er}^{3+}$ spins doped in a $\mathrm{Y}_{2} \mathrm{SiO}_{5}$ crystal using a high- $Q$, coplanar, superconducting resonator.
\end{abstract}

DOI: 10.1103/PhysRevB.84.060501

PACS number(s): 85.25.-j, 03.67.Lx, 42.50.Pq, 76.30.Kg

Quantum communication is a rapidly developing field of science and technology, which allows the transmission of information in an intrinsically secure way. ${ }^{1}$ As well as its classical counterpart, a quantum communication network can combine various types of systems which transmit, receive, and process information using quantum algorithms. ${ }^{2}$ For example, the nodes of such a network can be implemented by superconducting (SC) quantum circuits operated in the $\mathrm{GHz}$ frequency range, ${ }^{3}$ whereas fiber optics operated at near infrared can be used to link them over long distances. For the reversible transfer of quantum states between systems operating at $\mathrm{GHz}$ and optical frequency ranges, one must use a hybrid system. ${ }^{4}$ Spin ensembles coupled to a microwave resonator or to a SC qubit represent one of the possible implementations of such a system..$^{5-8}$ The collective coupling strength of a spin ensemble is increased with respect to a single spin by the square root of the number of spins. Transparent crystals doped with paramagnetic ions often possess long coherence times, ${ }^{9,10}$ and the collective coupling has been recently demonstrated with nitrogen-vacancy centers in diamond, ${ }^{11-13}$ organic molecules, ${ }^{14}$ and $\left(\mathrm{Cr}^{3+}\right)$ ions in ruby. ${ }^{12}$

In this Rapid Communication, we report on the ultralowpower electron-spin-resonance (ESR) spectroscopy of an erbium-ion spin ensemble at sub-Kelvin temperatures using a high- $Q$, coplanar, $\mathrm{SC}$ resonator. $\mathrm{The} \mathrm{Er}^{3+}$ ions are distinct from other spin ensembles due to their optical transition at the telecom $C$ band, i.e., inside the so-called erbium window at $1.54 \mu \mathrm{m}$ wavelength, and their long measured optical coherence time. ${ }^{15}$

The energy-level diagram of erbium ions embedded inside a crystal is shown in Fig. 1(a). The electronic configuration of a free $\mathrm{Er}^{3+}$ ion is $4 f^{11}$, with $\mathrm{a}^{4} I$ term. The spin-orbit coupling splits it into several fine structure levels. An optical transition at the telecom wavelength occurs between the ground state ${ }^{2 S+1} L_{J}={ }^{4} I_{15 / 2}$ and the first excited state ${ }^{4} I_{13 / 2}$, where $S$, $L$, and $J$ are the respective spin, orbital, and total magnetic momenta of the ion. The weak crystal field splits the ground state into eight $(J+1 / 2)$ Kramers doublets. ${ }^{16}$ At cryogenic temperature, only the lowest doublet $Z_{1}$ is populated, therefore the system can be described as an effective electronic spin with $S=1 / 2$. However, erbium has five even isotopes, ${ }^{162} \mathrm{Er},{ }^{164} \mathrm{Er}$, ${ }^{166} \mathrm{Er}$, ${ }^{168} \mathrm{Er}$, and ${ }^{170} \mathrm{Er}$, and one odd isotope, ${ }^{167} \mathrm{Er}$ (natural abundance $22.9 \%$ ) with a nuclear spin $I=7 / 2$. Therefore, the electronic states of ${ }^{167} \mathrm{Er}$ with effective spin projection $m_{S}= \pm 1 / 2$ are additionally split into eight hyperfine levels. ${ }^{17}$

The magnetic properties of erbium ions are associated with an unquenched total orbital moment $J$ in a crystal field, which results in the appearance of a large magnetic moment of nearly $7 \mu_{B}$, where $\mu_{B}$ is the Bohr magneton, at particular orientations of the applied magnetic field. ${ }^{18}$ The large spin tuning rate of $\sim 200 \mathrm{GHz} / \mathrm{T}$ makes erbium-doped crystals favorable for its integration with SC qubits that can be operated only at a relatively low magnetic field. Such an integrated spin-SC device can be very attractive for its applications particularly in quantum repeaters, where one can store quantum information and perform local operations. ${ }^{19}$ A quantum state of the optical field can be mapped into spin waves encoded in Zeeman or hyperfine levels of erbium ions ${ }^{20,21}$ and transferred later to the quantum state of the microwave field. ${ }^{4,6}$ The strong coupling between SC qubits and a microwave resonator allows fast quantum gate operations on the state of the microwave field at nanosecond time scales. ${ }^{22,23}$ This sequence can also be launched in the reverse order, thus establishing a coherent quantum transfer between $\mathrm{GHz}$ and optical frequency ranges.

In this experiment, we use a single $\mathrm{Y}_{2} \mathrm{SiO}_{5}$ crystal doped with $0.02 \%$ of $^{3+}$ (Er:YSO) (Scientific Materials, Inc.). The crystal has dimensions of $1 \times 1.5 \times 3 \mathrm{~mm}^{3}$ and it is glued on top of the silicon chip with a $\lambda / 2$ coplanar Niobium $\mathrm{SC}$ resonator; see Fig. 1(b). The resonance frequency of the rare-earth ion chip is $\omega_{0}=2 \pi \times 8.9 \mathrm{GHz}$ and its quality factor is $Q \approx 32000$ at the magnetic field of erbium transitions. The crystal orientation is shown in Fig. 1(c) with its optical extinction axes $b$ and $D_{1}$, and is specified by the angles $\theta$ and $\phi$ between these axes and the direction of the applied magnetic field. ${ }^{24}$ The $b$ axis of the crystal is directed along its $1.5 \mathrm{~mm}$ side and is perpendicular to the bias magnetic field $B\left(\theta=90^{\circ}\right)$ 
(a)
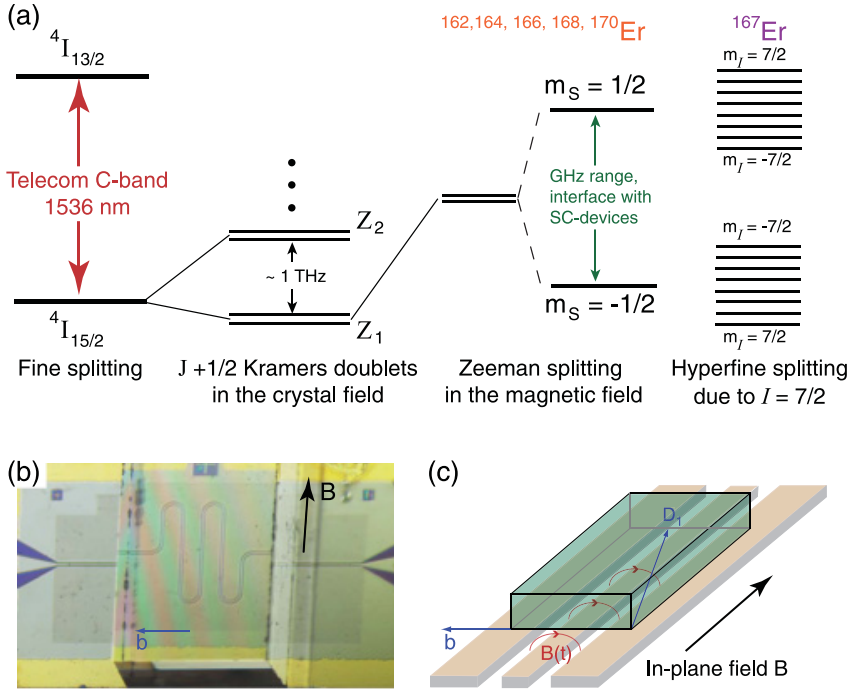

(c)

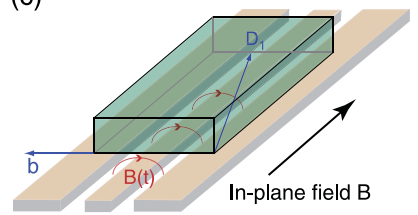

FIG. 1. (Color online) (a) The structure of the energy levels of $\mathrm{Er}^{3+}$ ions. (b) A picture of the rare-earth ion chip. The Er:YSO crystal of $1 \times 1.5 \times 3 \mathrm{~mm}^{3}$ size is placed on the $\lambda / 2$ resonator. The width of the central transmission line of the resonator is $20 \mu \mathrm{m}$; gaps are $9 \mu \mathrm{m}$. The magnetic field is applied along the chip surface. Several interference fringes are visible due to improper placement of the crystal (see text). (c) The sketch of the crystal orientation marked with its optical extinction axes $b$ and $D_{1}$ with respect to the directions of the bias magnetic field $B$ and microwave field $B(t)$.

applied parallel to the chip surface. The angle between the magnetic field and $D_{1}$ is $\phi=-60^{\circ}$. The particular orientation of our experiment is chosen to maximize the $g$-factor for the crystallographic site 1 (Refs. 17 and 24), which falls into our field-scanning range between 0 and $70 \mathrm{mT}$ and is relevant for our experiment. The gap between the bottom of the crystal and the chip surface is controlled by observing Newton's interference fringes and, with proper placement, 1-2 fringes are visible, yielding a gap of about $0.5 \mu \mathrm{m}$. The experiment was performed in a ${ }^{3} \mathrm{He}$ cryostat, at a base temperature of $280 \mathrm{mK}$

In quantum optics, the resonator-spin ensemble interaction is usually described by the Tavis-Cummings Hamiltonian. ${ }^{25}$ Provided that the number of photons in the resonator is much smaller than the number of spins $N$, a spin ensemble behaves as a harmonic oscillator coupled to a cavity. Nevertheless, the full theoretical description of the real experimental situation must also include a dependence on the inhomogeneous broadening shape, the crystal magnetic anisotropy, and its finite temperature. Such a theory is the subject of active research, ${ }^{25,26}$ thus we use a simplified phenomenological treatment of the observed effect. The rotating component of the magnetization of the spins produces an oscillatory magnetic field, which perturbs the inductance $L_{0}$ of the resonator. The new inductance $L^{\prime}=L_{0}(1+\chi \xi)$, where $\chi=\chi^{\prime}-i \chi^{\prime \prime}$ is the dynamic magnetic susceptibility of the ensemble, with $\chi^{\prime}$ and $\chi^{\prime \prime}$ as the dispersive and absorptive parts, respectively, and $\xi$ is a geometric factor describing the spin distribution across the mode and its coupling to the oscillating field. Thus, the resonance frequency of the rare-earth chip is $\omega_{0}^{\prime}=$ $\omega_{0} / \sqrt{1+\chi \xi}$. In the case of weak coupling $\chi \xi \ll 1$, we obtain the following equations for the resonator frequency $\omega_{0}^{\prime}$ and its decay rate $\kappa_{0}^{\prime}$ :

$$
\begin{aligned}
& \omega_{0}^{\prime}=\omega_{0}+\frac{v^{2}\left(\omega_{0}-\omega_{s}\right)}{\left(\omega_{0}-\omega_{s}\right)^{2}+\Gamma_{2}^{* 2}}, \\
& \kappa_{0}^{\prime}=\kappa_{0}+\frac{v^{2} \Gamma_{2}^{*}}{\left(\omega_{0}-\omega_{s}\right)^{2}+\Gamma_{2}^{* 2}},
\end{aligned}
$$

where $v$ is the coupling strength, $\omega_{s}$ is the Larmor frequency of the spin ensemble, and $\Gamma_{2}^{*}$ is its total linewidth; see Ref. 27 (chapter 2). The coupling strength $v$ can be expressed via the static susceptibility of the spin ensemble $\chi_{0}$ and reads as $v=\omega_{0} \sqrt{\chi_{0} \xi / 2}$. The substitution of the actual value of $\chi_{0}$ yields the final expression for the collective coupling strength $v=\tilde{g} \mu_{B} \sqrt{\mu_{0} \omega_{0} n \xi / 4 \hbar}$, where $\tilde{g}$ is an effective $g$ factor due to the magnetic anisotropy of the crystal and $n$ is the spin concentration. For the concentration of erbium electronic spins of $n_{S} \sim 10^{18} \mathrm{~cm}^{-3}$, and the effective $g$-factor in the plane perpendicular to the magnetic field of $\tilde{g} \sim 7$, and filling factor $\xi \sim 0.25$, we expect the coupling strength $v / 2 \pi \sim 60 \mathrm{MHz}$.

The microwave transmission spectroscopy of the rareearth ion chip as a function of the bias magnetic field is presented in Fig. 2. By using a vector network analyzer, we measured the $S_{21}$ parameter, which contains both the amplitude and the phase of the signal. The probing power at the input of the resonator is about $1 \mathrm{fW}$, corresponding to an excitation level of $\sim 100$ microwave photons in the SC resonator. The amplitude of the transmitted signal $\left|S_{21}\right|$ as a function of the magnetic field and probing frequency is presented in Fig. 2(a). The spectrum consists of the large dispersive cavity shift at a bias field of $B=55 \mathrm{mT}$ and is associated with the magnetic dipole transition between electronic states with $m_{S}= \pm 1 / 2$. A regular pattern of six dark interruptions is associated with the odd erbium isotope and is due to allowed hyperfine transitions between states with the equal nuclear spin projection $m_{I}=7 / 2,5 / 2, \ldots,-1 / 2,-3 / 2$. The states with $m_{I}=-5 / 2$ and $-7 / 2$ do not enter into our field-scanning range.

To understand the measured spectrum, we numerically diagonalize the spin Hamiltonian for paramagnetic ions in the crystal (see Refs. 17 and 27, chapter 3):

$$
H=\mu_{B} \mathbf{B} \cdot \mathbf{g} \cdot \mathbf{S}+\mathbf{I} \cdot \mathbf{A} \cdot \mathbf{S}+\mathbf{I} \cdot \mathbf{Q} \cdot \mathbf{I}-\mu_{n} \mathrm{~g}_{n} \mathbf{B} \cdot \mathbf{I},
$$

where $\mathbf{g}$ is the $g$-factor tensor, $\mathbf{A}$ is the hyperfine tensor, $\mathbf{Q}$ is the nuclear quadrupole tensor, $\mu_{n}$ is the nuclear Bohr magneton, and $g_{n}$ is the nuclear $g$-factor. The first term in the Hamiltonian presents an electronic Zeeman splitting, the second one describes hyperfine interactions, the third one is the quadrupole term, and the last one is the Zeeman splitting due to the nuclear spin. The values for the tensors have been taken from a previous ESR study of Er:YSO crystal. ${ }^{17}$ The resulting eigenspectrum is presented in Fig. 2(b). The position of each energy level is drawn as a function of the applied magnetic field, and the experimentally observed magnetic transitions are shown by arrows. For the even erbium isotopes, only the first term in the Hamiltonian (3) survives and that results in the strong magnetic transition between electronic-spin states, $m_{S}= \pm 1 / 2$, shown with the red arrow and marked by the 

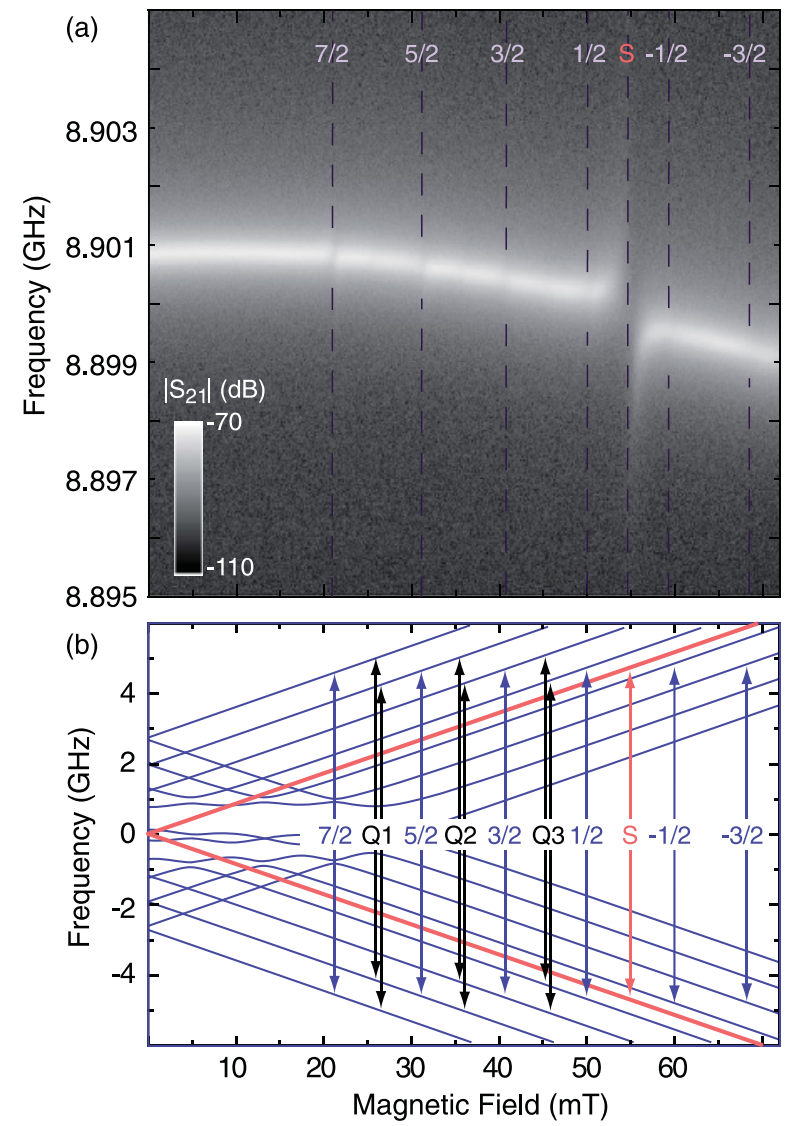

FIG. 2. (Color online) Transmission spectroscopy of the rareearth ion chip. (a) The amplitude of the transmitted signal $\left|S_{21}\right|$. The energy spectrum of different magnetic transitions is shown with dashed lines. The spin tuning rate $\gamma_{S} / 2 \pi=162.9 \mathrm{GHz} / \mathrm{T}$ and values for the hyperfine splitting are taken from the fit of the frequency shift (see text). (b) The calculated energy spectrum of Er:YSO in the magnetic field. Allowed and forbidden transitions are shown with arrows.

letter S. The odd ${ }^{167} \mathrm{Er}$ ion has eight allowed hyperfine transitions when $\Delta m_{I}=0$, which are shown by blue arrows and marked with $m_{I}$ numbers.

To extract the coupling strengths and linewidths for different transitions, each spectral line $\left|S_{21}\right|$ is fitted to a Lorentzian at every value of the magnetic field. The data corresponding to the shift of the resonator frequency $\omega_{0}^{\prime}$ for transitions $7 / 2$ and $\mathrm{S}$ are shown in Figs. 3(a) and 3(b). The dispersive behavior of the frequency shift in the vicinity of erbium spin transitions is well fit with Eq. (1). For the transition 7/2, the coupling strength extracted from that fit is $v_{7 / 2} / 2 \pi=2.1 \pm 0.3 \mathrm{MHz}$, which exceeds the decay rate of the resonator $\kappa / 2 \pi=$ $0.3 \mathrm{MHz}$. However, the linewidth of the spin ensemble exceeds the coupling strength and the same fit yields $\Gamma_{7 / 2}^{*} / 2 \pi=$ $70 \pm 1 \mathrm{MHz}$. The frequency shift of the resonator due to the coupling to the electronic-spin ensemble labeled S [Fig. 3(b)] has been studied for two excitation levels of $10^{2}$ (light gray dots) and $10^{5}$ (dark gray dots) microwave photons. The dashed line described by Eq. (1) fits well with the low-excitation spectrum, yielding $v_{S} / 2 \pi=11.6 \pm 0.2 \mathrm{MHz}$ and $\Gamma_{S}^{*} / 2 \pi=$ $145 \pm 5 \mathrm{MHz}$. At a high excitation level, the presented data correspond to a Dysonian line shape associated with spin
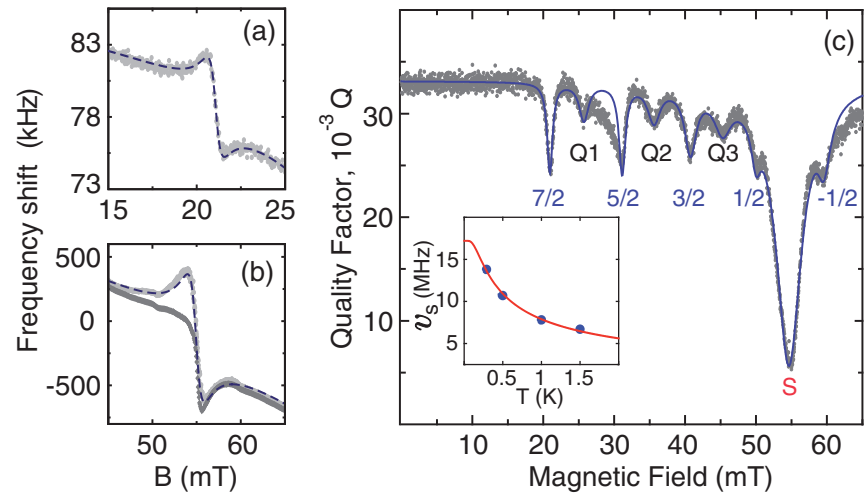

FIG. 3. (Color online) Frequency shift of the resonator $\omega_{0}^{\prime} / 2 \pi-$ $8.9 \mathrm{GHz}$ and its quality factor $Q$ vs. the applied magnetic field for (a) the transition 7/2 [Gray dots are experimental data. The dashed line is fit to Eq. (1)], and (b) the transition S [Light gray and dark gray dots are experimental data taken at the excitation level of $10^{2}$ and $10^{5}$ photons, respectively. The dashed line is a fit of the low-excitation data to Eq. (1)]. (c) Gray dots are experimental data points. The solid line is the fit of experimental data. Clearly observed dips correspond to different magnetic transitions. Inset: The temperature dependence of the collective coupling $v_{s}$ vs. temperature. Circles present measured data. The solid line is a fit to the data (see text).

diffusion in bulk metals. ${ }^{28}$ We believe that the observed mixture of the dispersive and absorptive line shapes might also be associated with spin diffusion in and out of the part interacting with the resonator mode.

The measured spectrum in Fig. 2(a) also reveals other interesting features: between the hyperfine transition, one can recognize an additional regular structure. To study this, we plot the quality factor of the resonator on Fig. 3(c) as a function of the magnetic field. The curve consists of a series of regular absorption dips originating from the magnetic coupling of electronic- and hyperfine-spin ensembles. It also contains an additional weak pattern appearing between the hyperfine transitions. We interpret these weak absorption lines as corresponding to the forbidden quadrupole transitions between the hyperfine states satisfying $\Delta m_{I}= \pm 1 .{ }^{17,27}$ These transitions are marked by the letters Q1, Q2, and Q3.

The experimental data of Fig. 3 can also be fit with $Q=\omega_{0} / \kappa_{0}^{\prime}$ by using Eq. (2), where each magnetic transitions contributes independently to the cavity decay rate $\kappa_{0}^{\prime}$. The data points between transitions Q1 and $5 / 2$, and after transition $-1 / 2$, are not fit well due to the presence of additional magnetic transitions related to the other crystallographic site. The fit of the quality factor behavior yields the following values: $v_{7 / 2} / 2 \pi=2.5 \pm 0.3 \mathrm{MHz}, \Gamma_{7 / 2}^{*} / 2 \pi=65 \pm 3 \mathrm{MHz}$, and $v_{S} / 2 \pi=13.8 \pm 0.1 \mathrm{MHz}, \Gamma_{S}^{*} / 2 \pi=141 \pm 3 \mathrm{MHz}$. The slight differences of the fitted parameters by using $\omega_{0}^{\prime}$ or $Q$ are probably due to the simplified model, which does not take into account the magnetic anisotropy of Er:YSO and inhomogeneous broadening of spin ensembles.

The coupling strength $v_{S}$ of the electronic-spin ensemble is found to vary as a function of its temperature. The transmission spectrum of the rare-earth chip has been taken at temperatures of $0.3,0.5,1.0$, and 1.5 Kelvin. The coupling strength $v_{S}$ is extracted from the change of the $Q$ and plotted as a function of the temperature in the inset of Fig. 3(c). The temperature 
dependence is fit to $v_{S}=v_{S}(0)\left[\tanh \left(\hbar \omega_{0} / 2 k_{B} T\right)\right]^{1 / 2}$ (see Ref. 29, chapter 11), and plotted with a solid line. The coupling strength of the ensemble at zero temperature, $v_{S}(0) / 2 \pi=$ $17.3 \pm 0.2 \mathrm{MHz}$, is the only fitted parameter.

We also found that linewidths of different spin ensembles are not the same and grow with the magnetic field. We explain this by a small misalignment of the crystal with respect to the magnetic field. When the angle $\theta$ deviates from $90^{\circ}$, the additional degeneracy due to the $C_{2}$ symmetry of the crystal is lifted and each erbium transition splits further into two different magnetic subclasses. ${ }^{17,18,24}$ Using the EASYSPIN package, ${ }^{30}$ we simulated ESR spectra of the Er:YSO crystal and bounded the maximum misalignment of the crystal to be $\Delta \theta<0.4^{\circ}$. Assuming that the observed effect is dominated by the magnetic class splitting, an additional contribution to the spin linewidth is estimated to be about $30 \mathrm{MHz}$. Such large linewidth cannot be completely attributed to the dephasing of spins. We measured the pure dephasing time using a Hahn echo sequence in a pulsed ESR spectrometer at the temperature of $7 \mathrm{~K}$, and found that for the $7 / 2$ transition, $T_{2}^{(7 / 2)} \approx 540 \mathrm{~ns}$, and for the $\mathrm{S}$ transition, $T_{2}^{(S)} \approx 200 \mathrm{~ns}$. Moreover, at the temperature of $0.3 \mathrm{~K}$, we would expect $T_{2}$ to be longer by at least an order of magnitude. ${ }^{9,10}$ Therefore, the additional contribution to the spin linewidths may be associated with field inhomogeneity or surface magnetism of the SC resonator. ${ }^{31}$
In conclusion, we have presented ESR spectroscopy of an $\mathrm{Er}^{3+}$ spin ensemble in $\mathrm{Y}_{2} \mathrm{SiO}_{5}$ crystal performed at the femtowatt excitation power level and at sub-Kelvin temperatures by using a SC coplanar resonator. Although the measured coupling strengths of different spin ensembles encoded in the Er:YSO crystal exceed the decay rate of the cavity, the strong coupling regime is not reached yet due to the large spin linewidths. The on-chip ESR spectroscopy allows us to resolve all the spin-state transitions of the $\mathrm{Er}^{3+}$ ions. The presented experiment realizes an important step toward the implementation of an interface between SC quantum circuits and optical quantum networks.

We thank M. Azarkh and M. Drescher for the ESR study of Er:YSO crystals, A. Abdumalikov and O. Astafiev (NEC) for the fabrication of the SC resonator, and C. Müller for his useful comments and critical reading of the manuscript. This work was supported by the CFN of DFG, the EU Projects MIDAS, SCOPE, and SOLID, and the BMBF Programm "Quantum Communications" through the project QUIMP. I.P. acknowledges the support of the Alexander von Humboldt Foundation. P.B. acknowledges the financial support of KIT and MWK of Baden-Württemberg through the RiSC Grant.
${ }^{1}$ N. Gisin and R. Thew, Nature Photon. 1, 165 (2007).

${ }^{2}$ J. Kimble, Nature (London) 453, 1023 (2008).

${ }^{3}$ J. Clarke and F. Wilhelm, Nature (London) 453, 1031 (2008).

${ }^{4}$ K. Tordrup, A. Negretti, and K. Mølmer, Phys. Rev. Lett. 101, 040501 (2008).

${ }^{5}$ J. Verdú, H. Zoubi, C. Koller, J. Majer, H. Ritsch, and J. Schmiedmayer, Phys. Rev. Lett. 103, 043603 (2009).

${ }^{6}$ A. Imamoğlu, Phys. Rev. Lett. 102, 083602 (2009).

${ }^{7}$ D. Marcos, M. Wubs, J. M. Taylor, R. Aguado, M. D. Lukin, and A. S. Sørensen, Phys. Rev. Lett. 105, 210501 (2010).

${ }^{8}$ J. Twamley and S. D. Barrett, Phys. Rev. B 81, 241202 (2010).

${ }^{9}$ S. Bertaina, S. Gambarelli, A. Tkachuk, I. N. Kurkin, B. Malkin, A. Stepanov, and B. Barbara, Nature Nanotechnol. 2, 39 (2007).

${ }^{10}$ S. Takahashi, R. Hanson, J. van Tol, M. S. Sherwin, and D. D. Awschalom, Phys. Rev. Lett. 101, 047601 (2008).

${ }^{11}$ Y. Kubo et al., Phys. Rev. Lett. 105, 140502 (2010).

${ }^{12}$ D. I. Schuster et al., Phys. Rev. Lett. 105, 140501 (2010).

${ }^{13}$ R. Amsüss et al., e-print arXiv:1103.1045 (accepted to Phys. Rev. Lett.).

${ }^{14}$ I. Chiorescu, N. Groll, S. Bertaina, T. Mori, and S. Myiashita, Phys. Rev. B 82, 024413 (2010).

${ }^{15}$ T. Böttger, C. W. Thiel, Y. Sun, and R. L. Cone, Phys. Rev. B 73, 075101 (2006).

${ }^{16}$ J. L. Doualan, C. Labbe, P. L. Boulanger, J. Margerie, R. Moncorge, and H. Timonen, J. Phys. Condens. Matter 7, 5111 (1995).
${ }^{17}$ O. Guillot-Noël, P. Goldner, Y. L. Du, E. Baldit, P. Monnier, and K. Bencheikh, Phys. Rev. B 74, 214409 (2006).

${ }^{18}$ I. N. Kurkin and K. P. Chernov, Physica B 101, 233 (1980).

${ }^{19}$ H. J. Briegel, W. Dur, J. I. Cirac, and P. Zoller, Phys. Rev. Lett. 81, 5932 (1998).

${ }^{20}$ L. Duan, M. D. Lukin, J. I. Cirac, and P. Zoller, Nature (London) 414, 413 (2001).

${ }^{21}$ B. Lauritzen, J. Minář, H. de Riedmatten, M. Afzelius, and N. Gisin, Phys. Rev. A 83, 012318 (2011).

${ }^{22}$ L. DiCarlo et al., Nature (London) 460, 240 (2009).

${ }^{23}$ R. C. Bialczak et al., Nature Phys. 6, 409 (2010).

${ }^{24}$ Y. Sun, T. Böttger, C. W. Thiel, and R. L. Cone, Phys. Rev. B 77, 085124 (2008)

${ }^{25}$ K. Henschel, J. Majer, J. Schmiedmayer, and H. Ritsch, Phys. Rev. A 82, 033810 (2010).

${ }^{26}$ Z. Kurucz, J. H. Wesenberg, and K. Mølmer, Phys. Rev. A 83, 053852 (2011).

${ }^{27}$ A. Abragam and B. Bleaney, Electron Paramagnetic Resonance of Transition Ions (Clarendon, Oxford, 1970).

${ }^{28}$ F. J. Dyson, Phys. Rev. 98, 349 (1955).

${ }^{29} \mathrm{C}$. Kittel, Introduction to Solid State Physics (Wiley, New Jersey, 2005).

${ }^{30}$ S. Stoll and A. Schweiger, J. Magn. Reson. 178, 42 (2006).

${ }^{31}$ S. Sendelbach, D. Hover, M. Muck, and R. McDermott, Phys. Rev. Lett. 103, 117001 (2009). 